Article

\title{
Numerical Investigation on Handling Stability of a Heavy Tractor Semi-Trailer under Crosswind
}

\author{
Qianwen Zhang ${ }^{1,2,3}$, Chuqi Su ${ }^{1,2,3}$, Yi Zhou ${ }^{1}$, Chengcai Zhang ${ }^{1, *}$, Jiuyang Ding ${ }^{4}$ \\ and Yiping Wang 1,2,3,* \\ 1 Hubei Key Laboratory of Advanced Technology for Automotive Components, \\ Wuhan University of Technology, Wuhan 430070, China; qianwenzhang@whut.edu.cn (Q.Z.); \\ suchuqi@whut.edu.cn (C.S.); yi.zhou.CP2@faw-vw.com (Y.Z.) \\ 2 Hubei Collaborative Innovation Center for Automotive Components Technology, \\ Wuhan University of Technology, Wuhan 430070, China \\ 3 Hubei Research Center for New Energy \& Intelligent Connected Vehicle, Wuhan University of Technology, \\ Wuhan 430070, China \\ 4 College of Automotive Engineering, Jilin University, Changchun 130025, China; jiuyang.ding@faw-vw.com \\ * Correspondence: zhangchc@whut.edu.cn (C.Z.); wangyiping@whut.edu.cn (Y.W.)
}

Received: 15 April 2020; Accepted: 23 May 2020; Published: 26 May 2020

\begin{abstract}
Due to the large lateral area of the trailer and variable road conditions, the handling stability of a heavy tractor semi-trailer under crosswind is very important for road safety. In this present work, numerical simulation is performed to study the crosswind effects on handling stability of a tractor semi-trailer. The aerodynamic characteristics of the tractor semi-trailer under different crosswind were computed by computational fluid dynamics (CFD). Then, mathematical models to reveal the relationship between the aerodynamic forces and crosswind were constructed to serve as inputs of the multi-body dynamics to analyze the handling stability under crosswind. The performance of crosswind stability is evaluated by the response of lateral acceleration, yaw rate and the lateral displacement. The lateral acceleration and yaw rate were decreased by a maximum of $14.6 \%$ and $16.5 \%$ compared to the truck without the deflector, which showed that the crosswind aerodynamics and stability were obviously improved.
\end{abstract}

Keywords: heavy tractor semi-trailer; crosswind stability; vehicle aerodynamic; dynamic characteristics; wind deflector

\section{Introduction}

In recent years, with the development of highways and logistics industries, more and more heavy tractor semi-trailers have been introduced into road transport. The crosswind stability, which is related to the safety of truck driving, has attracted wide concern. Due to its high center of gravity, narrow wheelbase (compared with the body height) and large area of container, the heavy tractor semi-trailer driving at high speed is more prone to lateral tilt and rollover when encountering strong crosswind [1]. Serious traffic accidents or strong psychological burden to the driver will be induced [2]. As shown in Figure 1, a sequence of scenes vividly displays some cases of wind-induced rollover of a tractor semi-trailer.

The study related to crosswind effect on vehicle performance could be basically classified into two aspects: one is crosswind aerodynamics [3], and the other is crosswind stability [4]. About crosswind aerodynamics [5], the influence of the crosswind on aerodynamic characteristics was mainly discussed, such as the fluctuation of the six aerodynamic components and the change of flow field. The most common evaluation methods for evaluating the aerodynamic performance of vehicles in 
different wind environments are road tests, static wind tunnel tests and simulations with varying yaw angles [6]. As for the crosswind stability, experimental and dynamic modeling simulation methods were adopted to investigate the dynamic response of the vehicle under crosswind, the deviation from the original driving path and the driver's handling of the vehicle. Some previous works have already concentrated on dynamic aerodynamics, which clearly demonstrate the importance of time-related changes under aerodynamic forces. When driving at high speed, the influence of aerodynamic force on vehicle performance is particularly important, especially under complex crosswind circumstances. For instance, Tural Tunay et al. [7] investigated the aerodynamic coefficients with different yaw angles on Ahmed vehicle by the wind tunnel test and Particle image velocimetry (PIV) measurements, which visualized the velocity flow field under the crosswind vividly and thoroughly revealed the underlying flow phenomena related to crosswind sensitivity of the ground vehicles. Gajendra et al. [8] discussed the crosswind aerodynamics of a sedan, and their research illustrated that the drag coefficient, lift coefficient and the yaw moment would increase with the growth of yaw angle and velocity of crosswind. Makoto et al. [9] analyzed a road vehicle's aerodynamic response in transient crosswinds. Cai et al. [10] proposed a framework to analyze a vehicle running on a long bridge in windy circumstances. On this basis, the aerodynamic response of vehicles running on highways or bridges can be analyzed and the critical wind speed can be evaluated to avoid potential accidents.

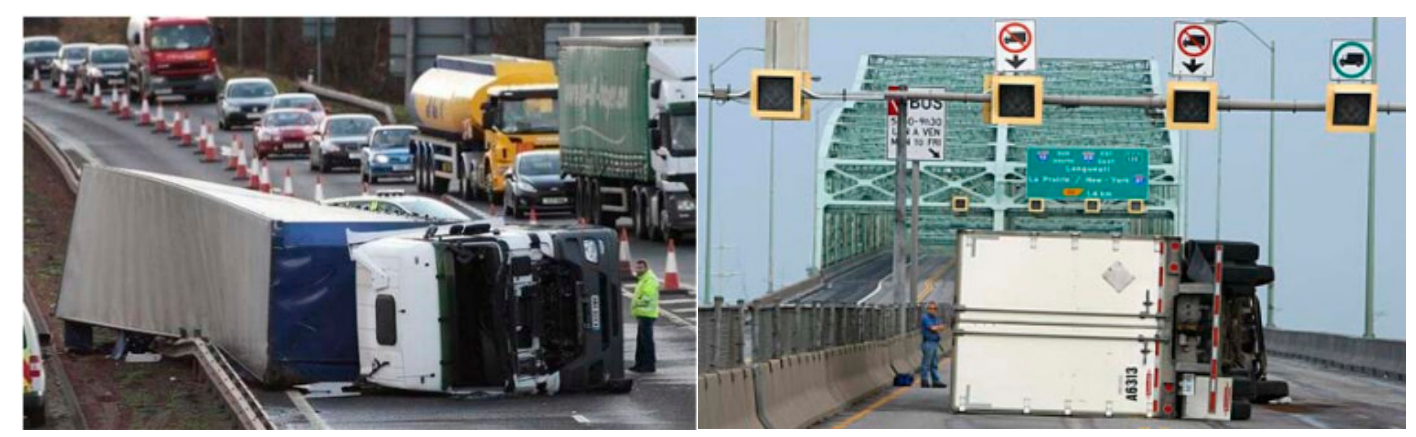

Figure 1. Wind-induced rollover of a tractor trailer.

In the aspect of crosswind stability, scholars have had extensive discussions on theoretical analysis, model simulation and experiments. Kee et al. [11] carried out an investigation on the riding stability of a passenger car under crosswind by the full-scale wind tunnel test and on-road test with a crosswind generator. Their results revealed the major parameter that affects the crosswind sensitivity of passenger cars was the yawing moment. In addition, high-speed stability would be improved with the reduction of lift force, lateral force and yawing moment. Fujihashi et al. [12] evaluated vehicle behavior under crosswind by using a full-vehicle dynamic simulation model with aerodynamic forces and moments, which were obtained by numerical simulation added on the gravity center. Nakashima [13] conducted vehicle dynamics simulations to analyze the effect of unsteady aerodynamics of a simplified truck under transient crosswinds. In his study, the horizontal motion of the vehicle was considered, and the vertical motion was assumed to be balanced statically. To investigate the influence of crosswind on directional deviation and handling performance, Juhlin et al. [14] constructed a gust wind model and applied to a parameterized bus. The results showed that the crosswind velocity was the main factor on directional deviation, and the yaw moment and lateral force coefficient were also the most significant considerations among the aerodynamic coefficients. The previous research showed that the research related to crosswind aerodynamics and crosswind stability were relatively separated from each other, which did not consider the interaction between the two parts [15].

It is undeniable that the crosswind will influence the aerodynamic characteristics and may induce lateral displacement. Moreover, by considering the change of aerodynamic characteristics under crosswind, the handling stability analysis is more convincing. Therefore, in this work, a coupling approach was adopted to investigate the crosswind aerodynamic and crosswind stability of a heavy 
duty tractor trailer, systematically. By this means, the aerodynamic characteristics under different crosswinds [16] were computed by computational fluid dynamics (CFD), and a mathematical model was constructed to describe the relationship between aerodynamic forces and crosswind. Then a full vehicle dynamic model of a tractor semi-trailer was constructed, and the effect of the crosswind on stability was investigated by considering the mathematical model of the aerodynamic force as an input.

Based on this coupling method, the heavy tractor trailer with and without a deflector was used to evaluate the aerodynamic characteristics and dynamic response of the truck in different crosswinds. The results have demonstrated that the deflector, which can reduce the lateral force and yawing moment, is also beneficial to improve the handling stability greatly under crosswind, and has a certain significance for engineering applications.

\section{Numerical Method and Validation}

\subsection{Governing Equations}

Computational fluid dynamics (CFD), which solves the coupling of the Reynolds averaged Navier-Stokes (RANS) equations to a turbulence model, has proven to be a very powerful and practical tool [17-21]. Two-equation models, such as $\kappa-\varepsilon$ [22], $\kappa-\omega$ [23] and the shear stress transport (SST) model [24], are the favorable options for CFD calculations. In the current work, the Renormalization Group (RNG) $\kappa-\varepsilon$ turbulence model [25] was selected to investigate the flow around the heavy truck, where the flow field induced was three-dimensional, turbulent and incompressible. It has been shown to better predict the separating flows and is widely used in engineering applications. For example, using two-equation models, previous scholars conducted research on street canyon flow [26], large tank fire $[27,28]$, internal combustion engine $[29,30]$ and heat exchange $[31,32]$. The corresponding governing equations are as follows:

Reynolds average continuity equation:

$$
\frac{\partial u_{i}}{\partial x_{i}}=0
$$

RANS equations:

$$
\frac{\partial u_{i}}{\partial t}+u_{j} \frac{\partial u_{i}}{\partial x_{j}}=-\frac{1}{\rho} \frac{\partial p}{\partial x_{i}}+\frac{1}{\rho} \frac{\partial}{\partial x_{j}}\left(\mu \frac{\partial u_{i}}{\partial x_{j}}-\overline{u_{i}^{\prime} u_{j}^{\prime}}\right)
$$

where $u$ and $u_{i}^{\prime}$ are mean and fluctuating velocities, respectively; subscripts $i, j=1,2,3$ stand for three different directions, $\mathrm{x}, \mathrm{y}$ and $\mathrm{z} ; \rho$ is the density, $p$ represents the mean pressure and $\mu$ denotes the dynamic viscosity.

The last terms of the right side of Equation (2) represent the Reynolds stress using the approach of the eddy viscosity principle after Boussinesq:

$$
-\overline{u_{i}^{\prime} u_{j}^{\prime}}=v_{t}\left(\frac{\partial u_{i}}{\partial x_{j}}+\frac{\partial u_{j}}{\partial x_{i}}\right)-\frac{2}{3} k \delta_{i j}
$$

where $v_{t}$ is the turbulence eddy viscosity, $k=\frac{1}{2} \overline{v_{i}^{\prime} v_{i}^{\prime}}$ is the turbulence kinetic energy and $\delta_{i j}$ is the Kronecker delta. The turbulence eddy viscosity could be computed by the function of turbulence kinetic energy $(k)$ and turbulence dissipation rate $(\varepsilon)$ :

$$
\begin{gathered}
v_{t}=C_{\mu} \frac{k^{2}}{\varepsilon} \\
\frac{\partial k}{\partial t}+u_{j} \frac{\partial k}{\partial x_{j}}=\frac{\partial}{\partial x_{j}}\left[\left(v+\frac{v_{t}}{\sigma_{k}}\right) \frac{\partial k}{\partial x_{j}}\right]+G-\varepsilon
\end{gathered}
$$




$$
\begin{gathered}
\frac{\partial \varepsilon}{\partial t}+u_{j} \frac{\partial \varepsilon}{\partial x_{j}}=\frac{\partial}{\partial x_{j}}\left[\left(v+\frac{v_{t}}{\sigma_{\varepsilon}}\right) \frac{\partial \varepsilon}{\partial x_{j}}\right]+C_{\varepsilon 1} \frac{\varepsilon}{k} G-C_{\varepsilon 2} \frac{\varepsilon^{2}}{k}-\frac{C_{\mu} \eta^{3}\left(1-\eta / \eta_{0}\right)}{1+\beta \eta^{3}} \frac{\varepsilon^{2}}{k} \\
G=-\overline{u_{i}^{\prime} u_{j}^{\prime}} \frac{\partial u_{i}}{\partial x_{j}}=v_{t}\left(\frac{\partial u_{i}}{\partial x_{j}}+\frac{\partial u_{j}}{\partial x_{i}}\right) \frac{\partial u_{i}}{\partial x_{j}}
\end{gathered}
$$

where $\eta=(k / \varepsilon)\left(G / v_{t}\right)^{0.5}, \sigma_{k}=\sigma_{\varepsilon}=0.7149$ is the turbulent Prandtl number in the $k$ and the $\varepsilon$ equation [33], $C_{\mu}=0.0845, C_{1 \varepsilon}=1.42, C_{2 \varepsilon}=1.68, \eta_{0}=4.38, \beta=0.012$.

The solutions of the governing equations were carried out in STAR-CCM+. The steady-state, implicit scheme was utilized in the incompressible flow solver. The governing equations were discretized by using a vertex centered unstructured finite volume method. The segregated solver was used in the computations. A second-order scheme was applied for the spatial discretization.

\subsection{Validation Case of Heavy Tractor Semi-Trailer Model}

The model studied was a full-scale heavy tractor semi-trailer (see Figure 2) with a length, width and height of 16,903, 2438 and $3988 \mathrm{~mm}$, respectively. Some details such as the engine room under the cabin, together with the radiator and other small parts around the engine, were ignored in the geometry by considering the complexities involved in mesh generation. A flat surface was added to the wheel surface adjacent to the ground, and the body surface was smoothed to save the computational resource.
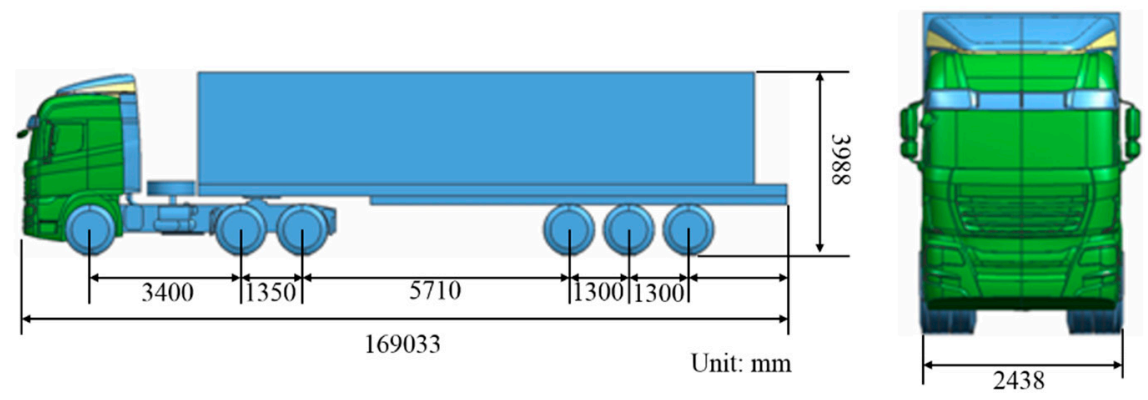

Figure 2. Validation model.

To validate the selection turbulence model, drag coefficient, lateral force coefficient, lift coefficient and yawing moment coefficient were compared with experimental results measured at the HD-2 Boundary Layer Wind Tunnel (HD-2 BLWT) at Hunan University and also the results from Gong [34]. The 1/6-scale truck model is used in the wind tunnel displayed in Figure 3. The numerical simulation model used in the current work is the same as that used by Gong and the experiment. The setup of the boundary conditions is also consistent with Gong's work. The boundary condition of the computational domain and grids resolution are delineated in Figures 4 and 5. Inlet 1 and inlet 2 are the velocity inlets, while outlet 1 and outlet 2 are set to the pressure outlet. In order to achieve the target of high precision, the domain was discretized by an unstructured trimmer grid in STAR-CCM+. In consideration of the large amount of separation around the tractor trailer, the resolution around the vehicle ranged from 25 to $40 \mathrm{~mm}$, and two refinement regions were applied around the vehicle.

The aerodynamic coefficients at different relative inflow angles are plotted in Figure 6 and show that the computational results are in agreement with the experimental and Gong's results. Among them, the aerodynamic lift coefficient obtained from the simulation is slightly different from the comparison experimental data and Gong's results, which may be caused by the difference between the ground clearance of the truck model in the numerical simulation and the wind tunnel test. In general, the selected turbulence model and computational setup could capture the three-dimensional, turbulent and incompressible flow field around the truck. 


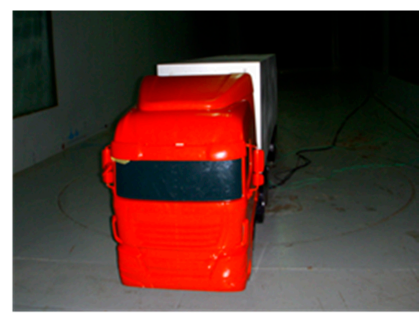

Figure 3. 1/6-scale wind tunnel test model.

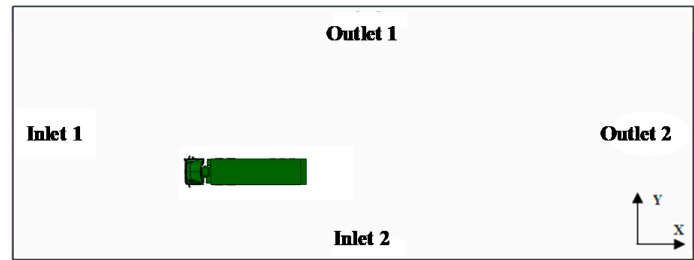

Figure 4. Boundary condition of computational domain.

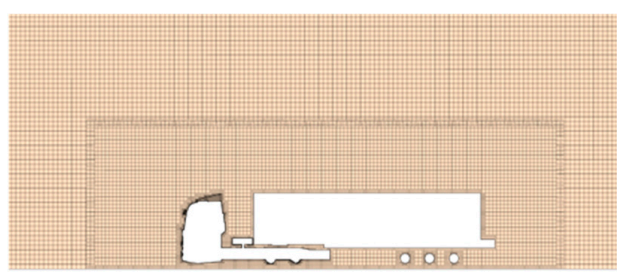

Figure 5. The longitudinal section of the grids.

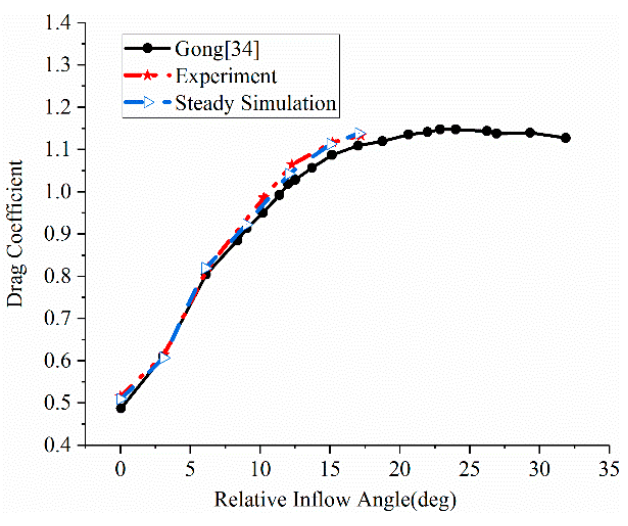

(a)

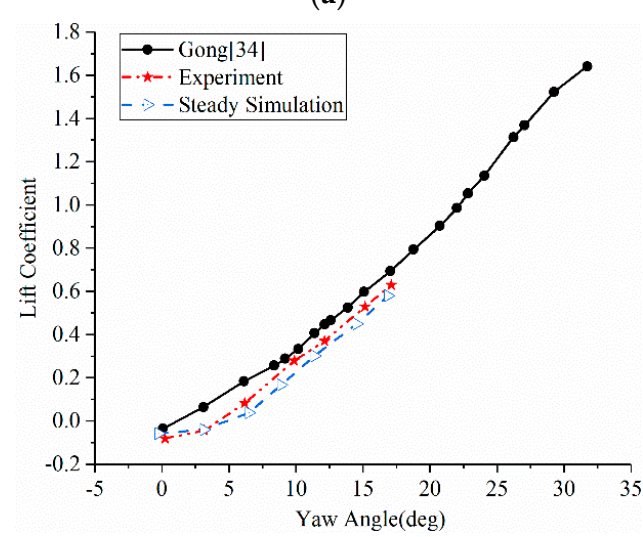

(c)

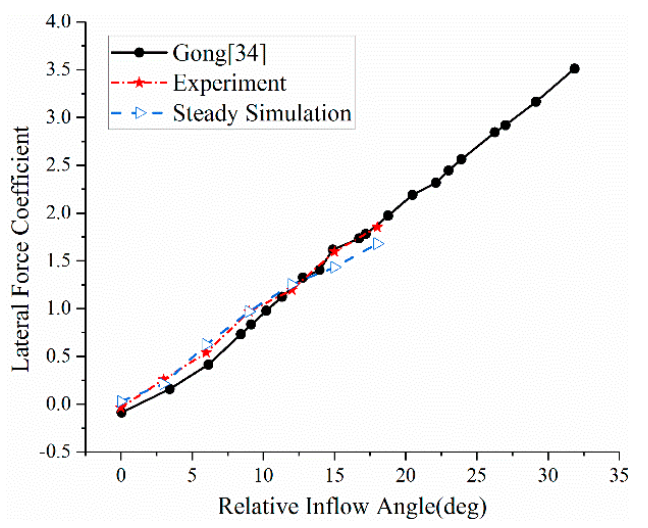

(b)

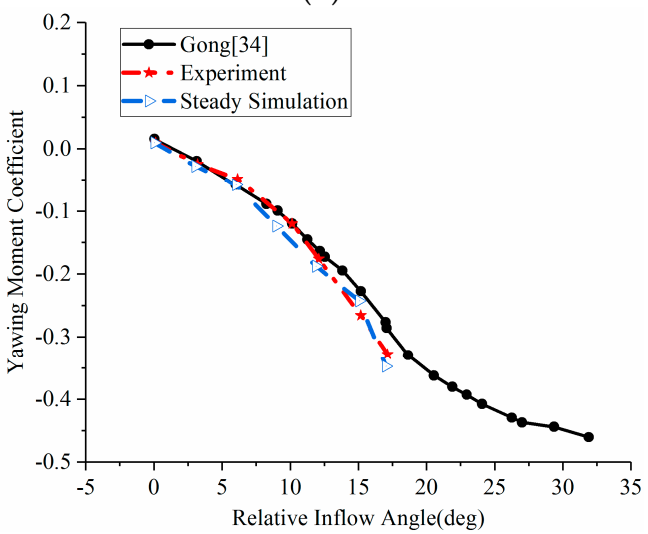

(d)

Figure 6. The comparison between experimental and computational results: (a) drag coefficient; (b) lateral force coefficient; (c) lift coefficient; (d) yawing moment coefficient. 


\section{Computation of the Aerodynamic Characteristics}

\subsection{Detailed Study of Target Vehicle Model}

The simulation for the target model was conducted on the same analysis region as the former one. The deflector has been removed from the original model, to study the influence of the additional device on the aerodynamic characteristics and handling stability of the heavy truck under the crosswind (see Figure 7).

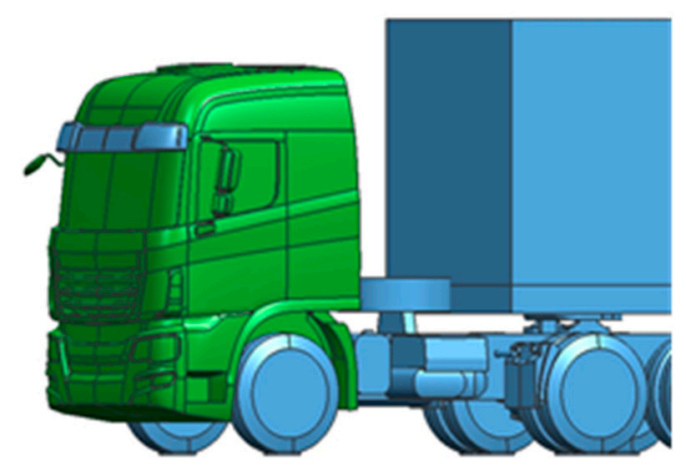

Figure 7. Target model.

\subsection{Computational Domain and Boundary Condition}

Turbulence model and calculation settings are the same as mentioned above. A large cuboid computational domain is illustrated in Figure 8; its length, width and height are 200, 37 and $20 \mathrm{~m}$, respectively. The blockage ratio with respect to cross section area of the vehicle was less than $0.25 \%$, which is satisfied with the typical requirement, less than 5\% [35]. The vehicle is about $3 \mathrm{~L}$ far away from the inlet and the side of the truck is about $5 \mathrm{~W}$ away from the crosswind inlet. The boundary condition setups are as follows: a resultant velocity $\left(V_{R}\right)$ whose direction is characterized by relative inflow angular $(\beta)$ is added at the freestream inlet and crosswind inlet. The x-component $\left(V_{1}\right)$, which is equivalent to the speed of vehicle, is set to $30 \mathrm{~m} / \mathrm{s}$. The $\mathrm{y}$-component $\left(V_{2}\right)$, which is equivalent to the speed of the crosswind, is changeable to simulate different crosswind intensities. Outlets were set as the pressure outlet, and the top of the computational domain was a free-slip wall so that they had no viscous effect on the results. The ground of the computational domain was set to a moving wall with the same velocity as that of the speed of the vehicle. The density of air is equivalent to $25^{\circ} \mathrm{C}$, $1.205 \mathrm{~kg} / \mathrm{m}^{3}$. Regarding the inlet velocity and vehicle length, the Reynolds number is $2.25 \times 10^{7}$. The upstream turbulence intensity was $0.5 \%$.

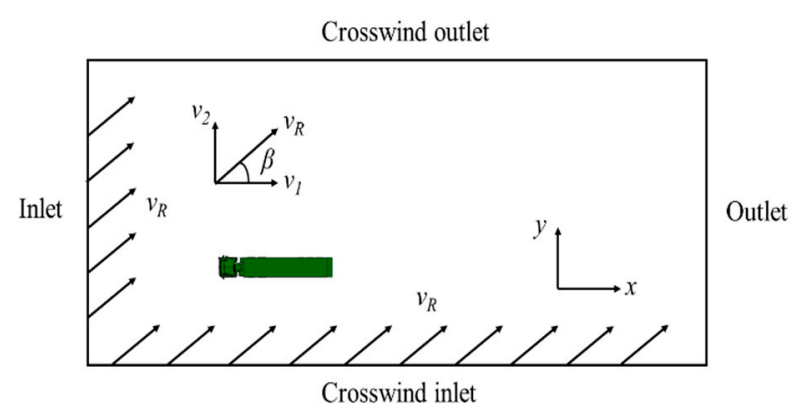

(a)

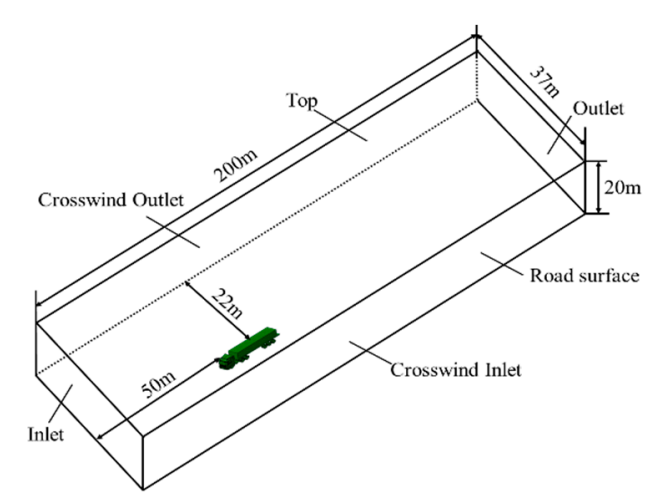

(b)

Figure 8. Computational domain: (a) top view; (b) isometric diagram. 


\subsection{Computational Grid}

The grids were hexahedron unstructured meshes generated in STAR-CCM+ (see Figure 9). To enhance the simulation accuracy, prismatic grids were generated near the vehicle surface as the boundary layer. Special attention was paid to the hook face in order to maintain the grid quality [36].

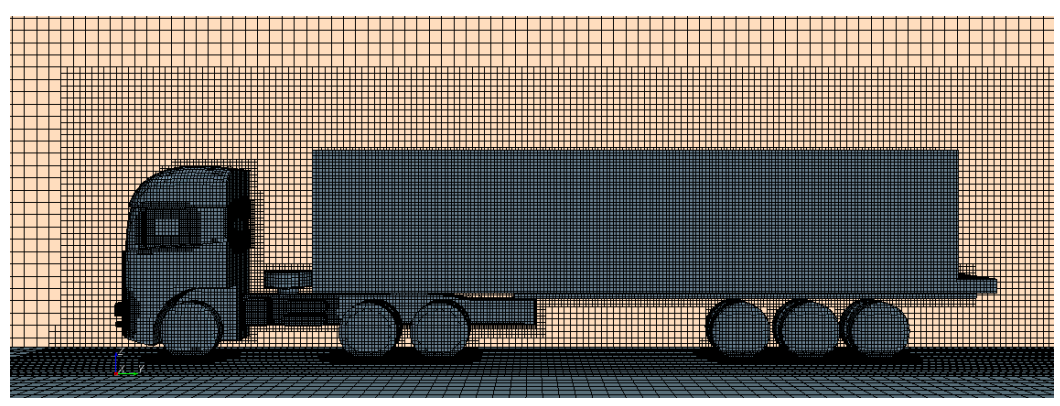

Figure 9. Mesh distribution.

Different computational grids were used to check the grid independence, which was carried out by three grid strategies, namely six million for the coarse mesh, eight million for the medium mesh and ten million for the fine mesh [37]. The drag coefficient was selected as the evaluation criterion. The results improved from the coarse mesh to medium mesh, and the drag coefficient was 0.6997 and 0.7045 for the medium and fine mesh, respectively; the deviation of the two grid strategies is approximately $0.69 \%$, which means, a negligible change was spotted from medium mesh to fine mesh. Considering the computational cost and efficiency, the medium mesh was used in current work.

\subsection{Results and Discussion}

Assuming the vehicle drives at a steady velocity, relative inflow angular orientation increases with the growth of crosswind. The crosswind intensities change from zero level to the seventh level of the Beaufort scale, which means the relative inflow angle varies from 0 to 21 degrees.

Aerodynamic forces and moments on a road vehicle include drag $F_{D}$, lift $F_{L}$, lateral force $F_{S}$, rolling moment $M_{R}$, pitching moment $M_{P}$ and yawing moment $M_{Y}$. The definitions and positive directions of the forces and moments are shown in Figure 10. These forces and moments refer to the gravity center of the vehicle in the present work. The expressions for aerodynamic coefficients are as follows:

$$
\begin{gathered}
C_{D}=\frac{F_{D}}{\frac{1}{2} \rho V^{2} A} \\
C_{L}=\frac{F_{L}}{\frac{1}{2} \rho V^{2} A} \\
C_{S}=\frac{F_{S}}{\frac{1}{2} \rho V^{2} A} \\
C_{R M}=\frac{M_{R}}{\frac{1}{2} \rho V^{2} A L} \\
C_{P M}=\frac{M_{P}}{\frac{1}{2} \rho V^{2} A L} \\
C_{Y M}=\frac{M_{Y}}{\frac{1}{2} \rho V^{2} A L}
\end{gathered}
$$

in which $V, A$ and $L$ are the relative speed, the frontal project area $\left(A=9.665 \mathrm{~m}^{2}\right.$ in the current work) and the wheelbase of the vehicle ( $L=13.1 \mathrm{~m}$ in the current work). 


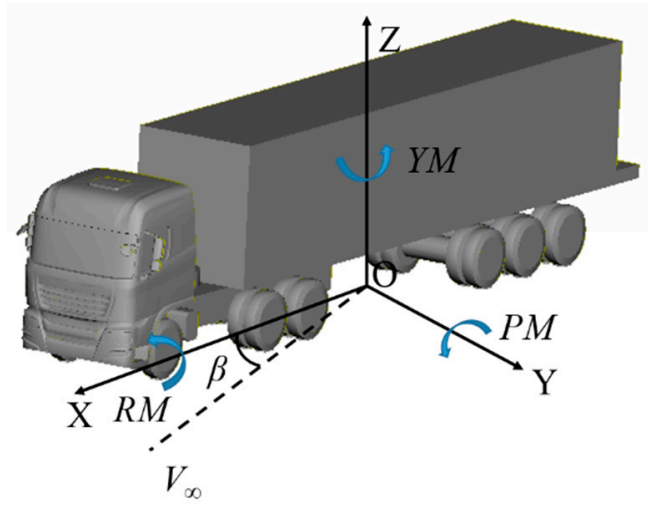

Figure 10. Aerodynamic forces and moments on the vehicle.

By changing the direction and value of the relative speed, the aerodynamic forces and moments can be simulated under different speeds and crosswind strength. The origin of coordinate in this numerical simulation is located at the projection of the center point between the third axis and the fourth axis of the container truck on the ground. The variation of the aerodynamic coefficients with relative inflow angles plotted in Figure 11 reveals that all aerodynamic coefficients trend to increase with these relative inflow angles, and the lateral force, and rolling and yawing moment, are the most sensitive to the relative inflow angle. In addition, the lateral force, yawing moment and rolling moment are the main causes of side-slip and rollover accidents. The lift and pitching moment may reduce the grip of tyres and thus are harmful for driving safety. It is obvious that the yawing moment and rolling moment increased notably with the increase of the crosswind speed. Previous studies [24] have shown that yawing moment made a greater impact on the handling stability, but the specific effects on the performance need to be investigated further in the dynamic simulation.

To identify the mathematical relationship of aerodynamic characteristics under crosswind, and couple with crosswind stability analysis, a polynomial was used to fit the variation of the aerodynamic coefficient with relative inflow angles. The polynomial models for the curves were given by:

$$
y=\sum_{i=1}^{n+1} p_{i} x^{n+1-i}
$$

in which $n+1$ is the order of the polynomial, $n$ is the degree of the polynomial. The order gives the number of coefficients to be fit, and the degree gives the highest power of the predictor variable. In the present work, a two-order polynomial was adopted to get the polynomial model of the aerodynamic coefficients. To evaluate the fitting precision, $R$-square was introduced. $R$-square is defined as the ratio of the sum of squares of the regression (SSR) and the total sum of squares (SST); the value of $R$-square closer to one indicates that the model accounts for a greater proportion of variance.

$$
\begin{gathered}
S S R=\sum_{i=1}^{n} \omega_{i}\left(\hat{y}_{i}-\bar{y}\right)^{2} \\
S S R=\sum_{i=1}^{n} \omega_{i}\left(y_{i}-\bar{y}\right)^{2} \\
R-\text { square }=\frac{S S R}{S S T}
\end{gathered}
$$




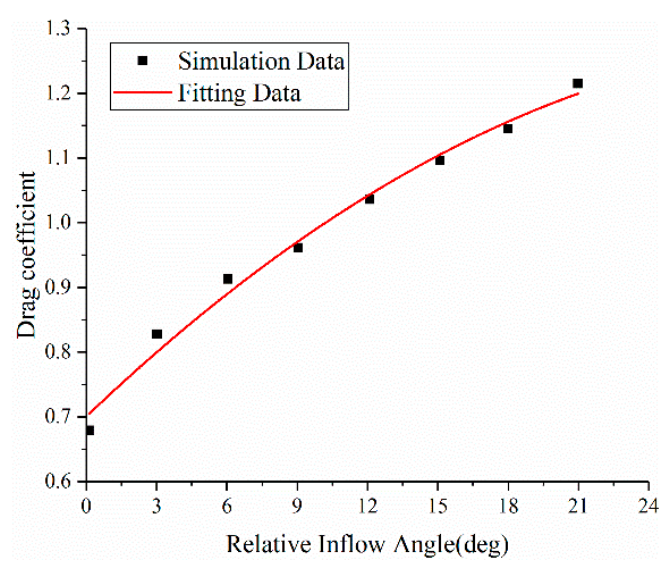

(a)

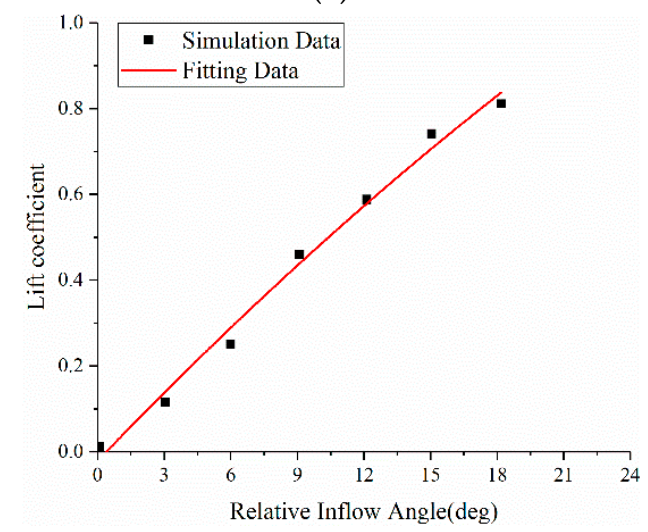

(c)

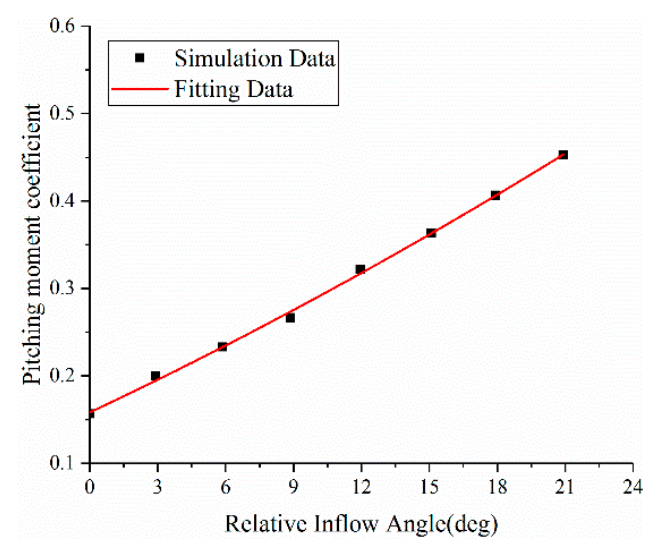

(e)

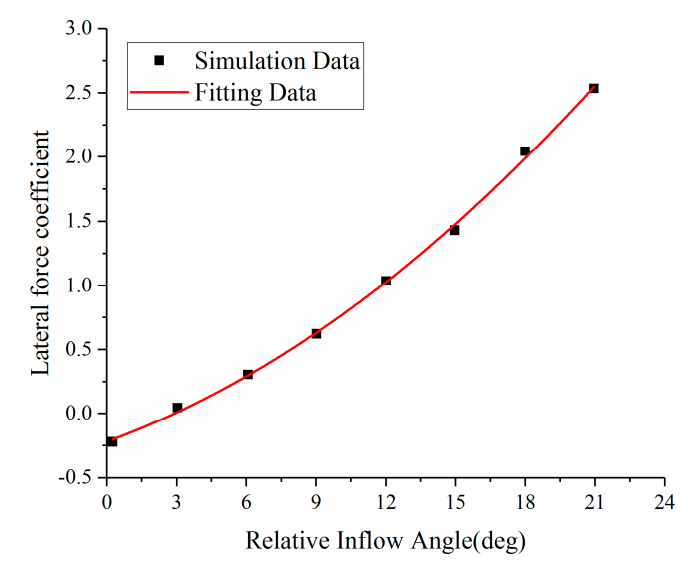

(b)

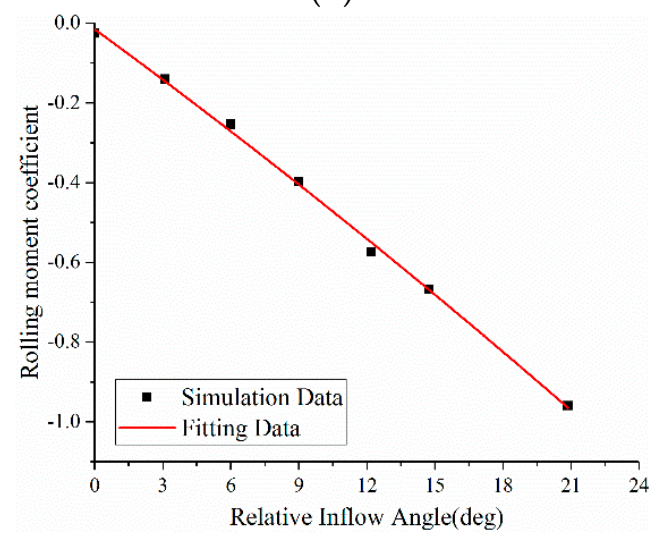

(d)

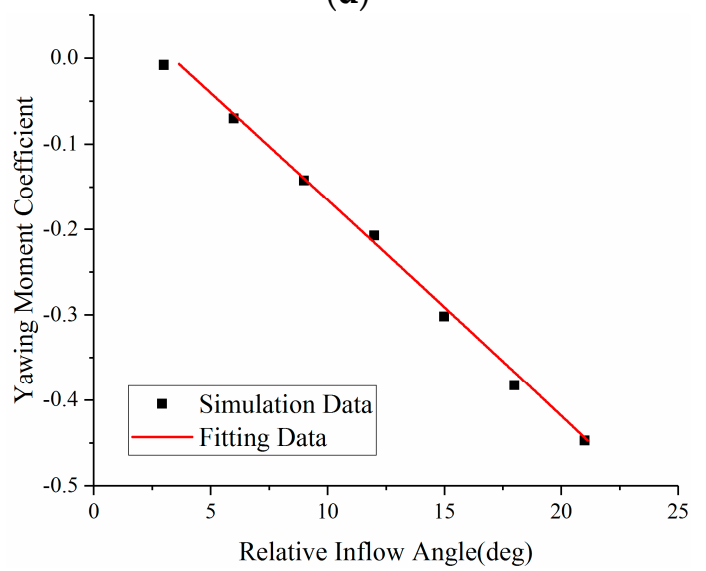

(f)

Figure 11. The variation curve of the aerodynamic coefficients to the relative inflow angles: (a) drag coefficient; (b) lateral force coefficient; (c) lift coefficient; (d) rolling moment coefficient; (e) pitching moment coefficient; (f) yawing moment coefficient.

The error analysis showed that the R-square for all fitting curves was larger than 0.95, which indicated good fitting accuracy. The fitting curves of aerodynamic coefficients compared with the simulation data were also shown in Figure 11, and the fitting formulas are as follows:

Aerodynamic drag coefficient:

$$
C_{D}=-0.00051632 \beta^{2}+0.03461 \beta+0.70049
$$


Aerodynamic lateral force coefficient:

$$
C_{S}=0.00315 \beta^{2}+0.06589 \beta-0.21907
$$

Aerodynamic lift coefficient:

$$
C_{L}=-0.00036945 \beta^{2}+0.05396 \beta-0.02155
$$

Aerodynamic rolling moment coefficient:

$$
C_{R M}=-0.000189227 \beta^{2}-0.04155 \beta-0.01531
$$

Aerodynamic pitching moment coefficient:

$$
C_{P M}=0.000097165 \beta^{2}+0.01218 \beta+0.15676
$$

Aerodynamic yawing moment coefficient:

$$
C_{Y M}=-0.00002360 \beta^{2}-0.0246 \beta+0.08322
$$

Figure 12 shows the 3D streamlines around the semi-trailer truck at different crosswinds. It can be seen that there was a low velocity region at the front of the tractor as the air tended to stagnate, followed by acceleration to high velocity at the separation point on the corner of the tractor. As the intensity of the crosswind increases from zero level to the seventh level, the flow field around the tractor semi-trailer becomes more and more asymmetric. There are some complex vortex areas in the gap between tractor and trailer as well as at the back of the tractor, and with the increase of the crosswind, the vortex center moves from the windward side to the leeward side. The flow velocity around the tractor semi-trailer is gradually increased from the windward side to the leeward side, which will induce great increases in the lateral force and the yawing moment coefficient. The high velocity area between the tractor and the trailer becomes larger and rotated at the back of the tractor with the increase of the crosswind. The wake becomes asymmetric and the flow velocity increases from the windward side to the leeward side. The surface directly exposed to the crosswind showed generally lower velocity than the opposite side.

Figure 13 shows the distributions of pressure under different intensity crosswinds on the lateral surface of the heavy truck. On the windward side, the stagnation point shifts to the front corner of the tractor and the high-pressure zone around the stagnation point moves in the same direction. The stagnation point reaches to the front corner at zero level and transits to the lateral side of the tractor with an intensity increase of the crosswind. The high-pressure region spread to the lateral surface of the trailer at higher levels. On the opposite side, the airflow separates from the front corner of the tractor and the region of separation increases with the intensity increase of the crosswind. The separation also increases the asymmetry of the flow field. The low-pressure area enlarges with the intensity of the crosswind increasing. At the same instant, the low-pressure region on the lateral surface of the tractor and the trailer spreads to the rear section of the truck, with the intensity of the crosswind increasing. Hence, an asymmetrical pressure distribution first occurs around the front of the tractor and then spreads toward the rear section. With the increase of lateral pressure and the movement of the pressure center, the rolling moment coefficient and yawing moment coefficient increase monotonously. Regarding the drag, the balance between the high-pressure zone at the front of the tractor and the low-pressure zone at the rear of the trailer also changes with the crosswind due to the expansion of the recirculation region and the shift of the stagnation point. 


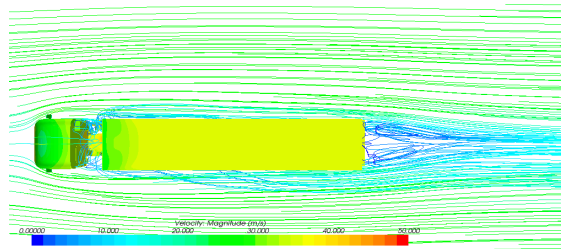

(a)

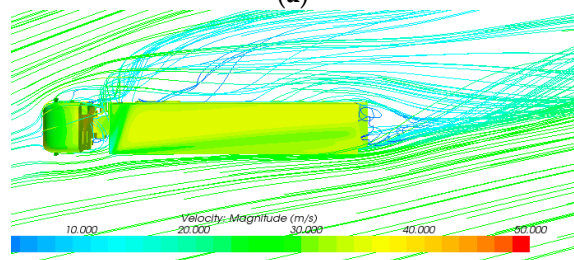

(c)

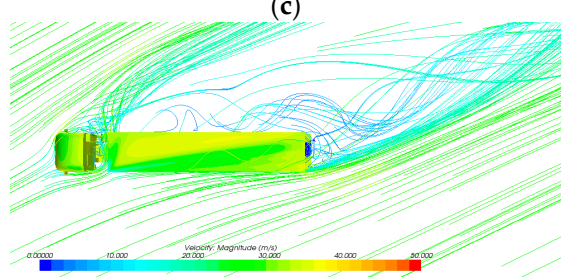

(e)

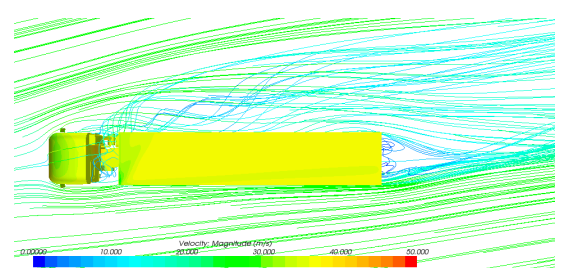

(b)

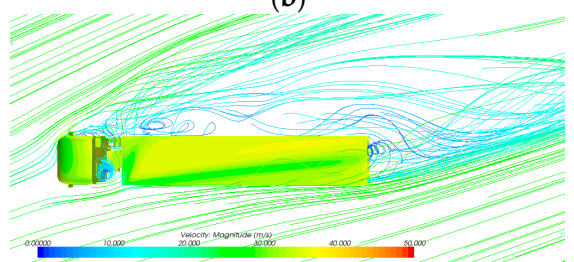

(d)

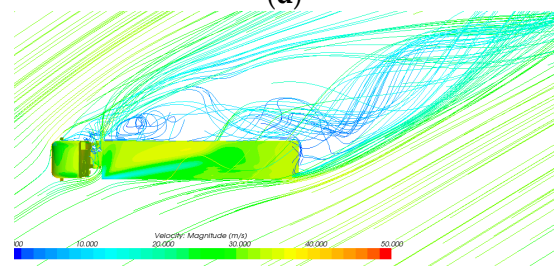

(f)

Figure 12. The flow field around the tractor semi-trailer at different intensities of crosswind: (a) zero level; (b) the third level; (c) the fourth level; (d) the fifth level; (e) the sixth level; (f) the seventh level.

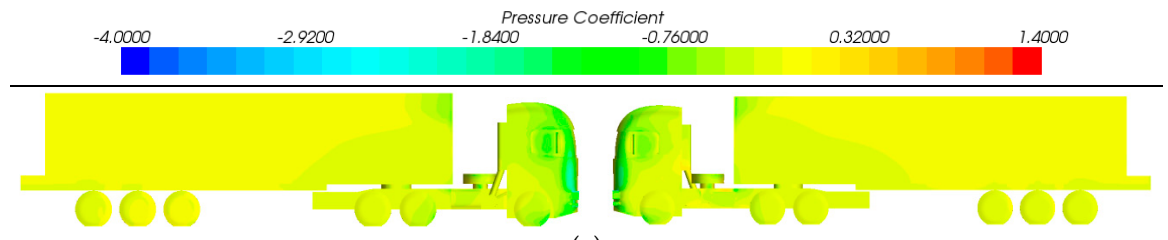

(a)
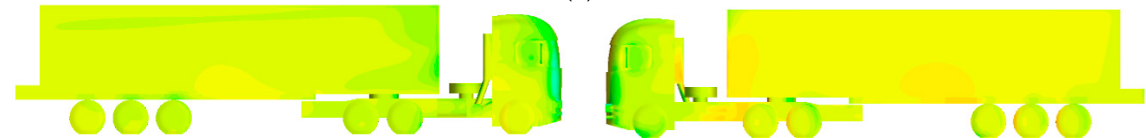

(b)
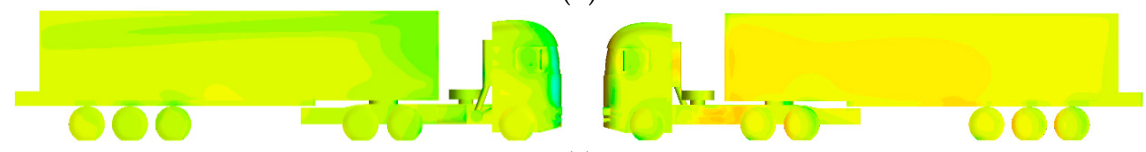

(c)
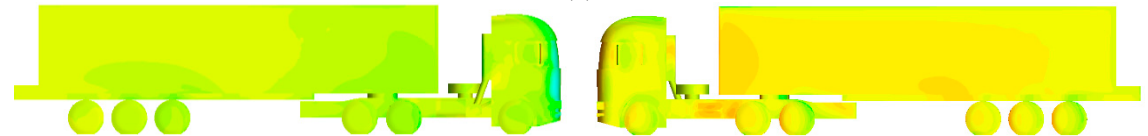

(d)
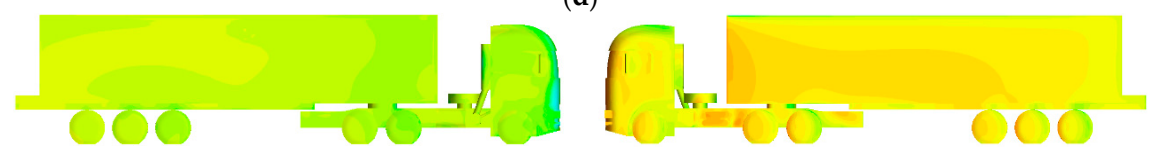

(e)
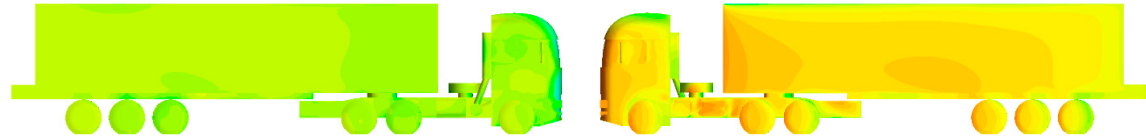

(f)

Figure 13. The distributions of pressure on the lateral surface at different crosswinds (left: leeward side, right: windward side): (a) zero level; (b) the third level; (c) the fourth level; (d) the fifth level; (e) the sixth level; (f) the seventh level. 


\section{Multi-Body Dynamic Simulation}

Previous research has studied the aerodynamic forces and moments under steady conditions, and just estimated the aerodynamic changes when the vehicles generated a yaw slip [38]. However, these aerodynamic characteristics will have some effects on the vehicle dynamics. Therefore, in this work, a coupling method that combined the changing aerodynamic coefficients with the vehicle dynamics system was used to analyze the effect of crosswind on the handling stability of a tractor semi-trailer.

A six-degrees-of-freedom (6-DOF) multi-body dynamic model of a six-axle tractor semi-trailer was constructed in the software TruckSim showed in Figure 14. The model consists of an aerodynamics module, vehicle body, driving system, steering system, braking system, power transmission system and so on. Some specifications of the tractor semi-trailer such as moment of inertia and center of gravity (c.g.) are described in Table 1. The tractor and trailer are treated as a single rigid body. In order to analyze the dynamic characteristics while considering the change of the aerodynamics under crosswind, the aerodynamic module is described by the fitting curves of the aerodynamic coefficients.

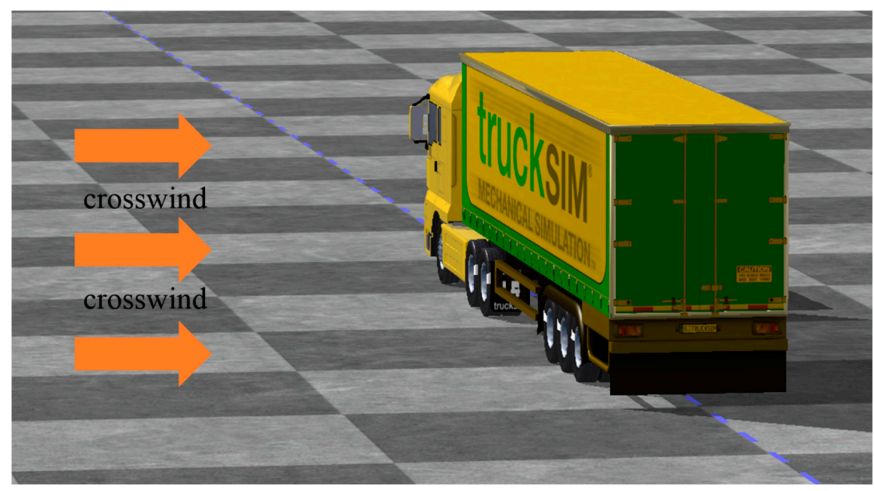

Figure 14. The schematic of multi-body dynamics model.

Table 1. Main parameters for the tractor semi-trailer.

\begin{tabular}{ccc}
\hline & Parameters & Values \\
\hline$m$ & Vehicle total mass (powered unit) & $7600 \mathrm{~kg}$ \\
$m_{s}$ & Vehicle sprung mass (powered unit) & $4455 \mathrm{~kg}$ \\
$l_{f}$ & Distance between c.g. and the 1-axis (powered unit) & $2.0 \mathrm{~m}$ \\
$l r$ & Distance between c.g. and the 3-axis (powered unit) & $2.75 \mathrm{~m}$ \\
$L$ & Vehicle length (powered unit) & $7.25 \mathrm{~m}$ \\
$H$ & Vehicle height (powered unit) & $3.9 \mathrm{~m}$ \\
$I_{x x}$ & Rolling moment of inertia (powered unit) & $2283.9 \mathrm{~kg} \bullet \mathrm{m}^{2}$ \\
$I_{y y}$ & Pitching moment of inertia (powered unit) & $35,402.8 \mathrm{~kg} \bullet \mathrm{m}^{2}$ \\
$I_{z z}$ & Yawing moment of inertia (powered unit) & $34,802.6 \mathrm{~kg} \bullet \mathrm{m}^{2}$ \\
$m_{t}$ & Vehicle total mass (trailer unit) & $7570 \mathrm{~kg}$ \\
$m_{t s}$ & Vehicle sprung mass (trailer unit) & $5500 \mathrm{~kg}$ \\
$l_{f t}$ & Distance between c.g. and the 3-axis (trailer unit) & $3.3 \mathrm{~m}$ \\
$l_{r t}$ & Distance between c.g. and the 6-axis (trailer unit) & $5.01 \mathrm{~m}$ \\
$L_{t}$ & Vehicle length (trailer unit) & $12.2 \mathrm{~m}$ \\
$H_{t}$ & Vehicle height (trailer unit) & $4.2 \mathrm{~m}$ \\
$I_{x x t}$ & Rolling moment of inertia (trailer unit) & $8997.1 \mathrm{~kg} \bullet \mathrm{m}^{2}$ \\
$I_{y y t}$ & Pitching moment of inertia (trailer unit) & $150,000 \mathrm{~kg} \bullet \mathrm{m}^{2}$ \\
$I_{z z t}$ & Yawing moment of inertia (trailer unit) & $150,000 \mathrm{~kg} \bullet \mathrm{m}^{2}$ \\
$K_{s f}$ & Upper vertical spring stiffness & $0.25 \mathrm{~N} / \mathrm{m}$ \\
$K_{s r}$ & Lower vertical spring stiffness & $0.7 \mathrm{~N} / \mathrm{m}$ \\
$C_{f}$ & Upper vertical damping & $15,000 \mathrm{Ns} / \mathrm{m}$ \\
$C_{r}$ & Lower vertical damping & $30,000 \mathrm{Ns} / \mathrm{m}$ \\
$C_{\alpha f}$ & Cornering stiffness of one tyre (front) & $140,000 \mathrm{~N} / \mathrm{rad}$ \\
$C_{\alpha r}$ & Cornering stiffness of one tyre (rear) & $140,000 \mathrm{~N} / \mathrm{rad}^{2}$ \\
\hline & &
\end{tabular}

The proposed tractor semi-trailer is equipped with a $300 \mathrm{~kW}$ diesel internal engine model, a torque transfer, a transmission, a transfer case and some differentials. The brake actuator of each wheel has been simply modeled with no braking pressure, and in order to simulate an open-loop steer control, the shifting control of the vehicle was clutch disengaged. The aerodynamic model is 
composed of the six aerodynamic coefficients; the force and moment vector acting on the sprung mass represent the aerodynamic effects. The aerodynamic characteristics will be changed when the aerodynamic parameters are different [39]. Finally, a multi-body dynamic model with given aerodynamic properties was established. The aerodynamic coefficients of the vehicle will be changed with the motion dynamically, and the change will have an important influence on the vehicle system dynamics. In the simulation, the driving speed was set to $30 \mathrm{~m} / \mathrm{s}$, and a crosswind with different strengths was added in the vertical direction of the vehicle driving direction.

To some degree, the lateral displacement, lateral acceleration and yaw rate could evaluate the handling stability of the tractor semi-trailer under crosswind. Figure 15 shows the variation of lateral displacement, lateral acceleration and yaw rate with time under different crosswinds. In order to obtain the dynamic response of the vehicle more clearly and accurately, the simulation time is set to $10 \mathrm{~s}$. In general, when the crosswind speed increases from 0 to $16.7 \mathrm{~m} / \mathrm{s}$, the lateral displacement, lateral acceleration and yaw rate also increase gradually. Due to the rise of the lateral force coefficient and the yawing moment coefficient, the growth rate of lateral displacement increases, and the variation gradient of lateral displacement also agrees well with the trends of the lateral coefficient and the yawing moment coefficient (see Figure 15a). Because the dynamic model is an open-loop, the lateral displacement of the tractor semi-trailer will increase continually. As to the lateral acceleration, it will increase to the maximum instantly once the vehicle encounters a crosswind, and reduces gradually then. Because the effective area of crosswind decreases with the tractor semi-trailer deviated from its original path, the lateral force and yawing moment also decrease so that the lateral acceleration decreases (see Figure 15b). The yaw rate shows a similar trend with the lateral acceleration. Previous studies [40] have indicated that the yaw rate is the most important factor on the handling stability, and the greater the yaw rate is, the worse the crosswind stability is.

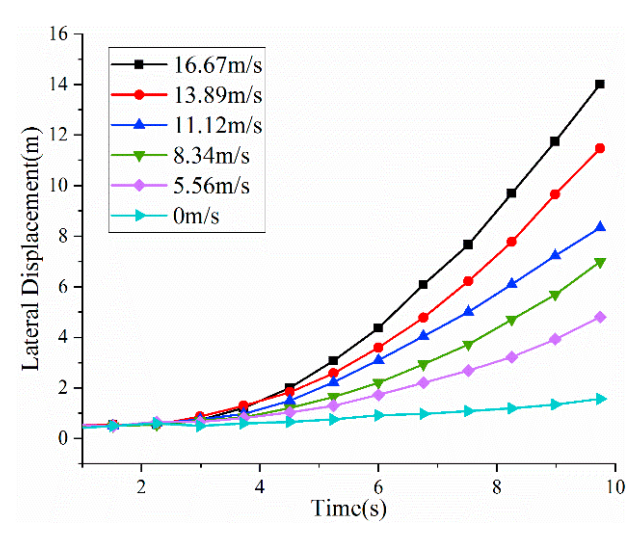

(a)

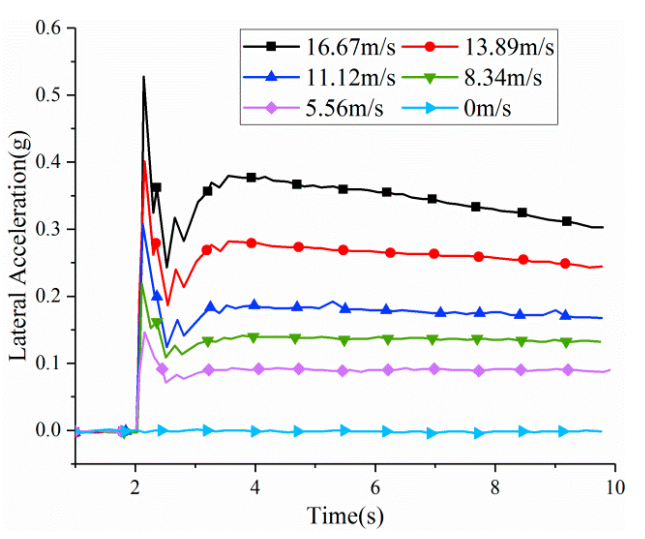

(b)

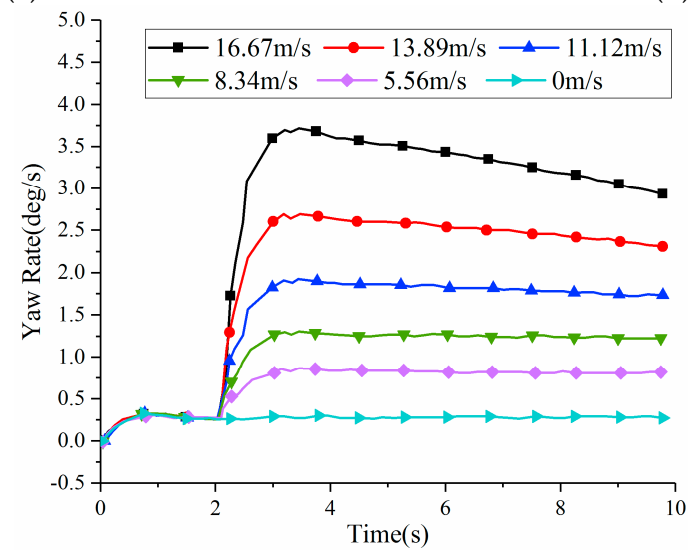

(c)

Figure 15. The evaluation indexes of crosswind stability at different crosswinds: (a) lateral displacement; (b) lateral acceleration; (c) yaw rate. 


\section{Effect of the Wind Deflector on Crosswind Stability}

In current research, the crosswind stability of tractor semi-trailer with and without a wind deflector has been investigated. The aerodynamic coefficients of the tractor semi-trailer with wind deflector under different yaw angles are obtained based on the above computational process. The variation tendency of the rolling moment coefficient and yawing moment coefficient is drawn in Figures 16 and 17. It is obvious that the rolling moment coefficient decreased when the wind deflector was added, which exerted great improvement on the performance of the vehicle. The wind deflector is also beneficial to reduce the yawing moment coefficient under crosswind, especially to the large relative angles, and that will have a positive impact on the handling stability of the vehicle. Obviously, this wind deflector could improve the aerodynamic characteristics of the vehicle in the crosswind circumstance. Meanwhile, the improvement of the aerodynamic characteristics would have large effects on the handling stability of vehicle. To investigate the improvement effects of the aerodynamic characteristics on handling stability under crosswind, the fitting formulas of aerodynamic characteristics of the vehicle with a wind deflector are also applied to the multi-body dynamic model.

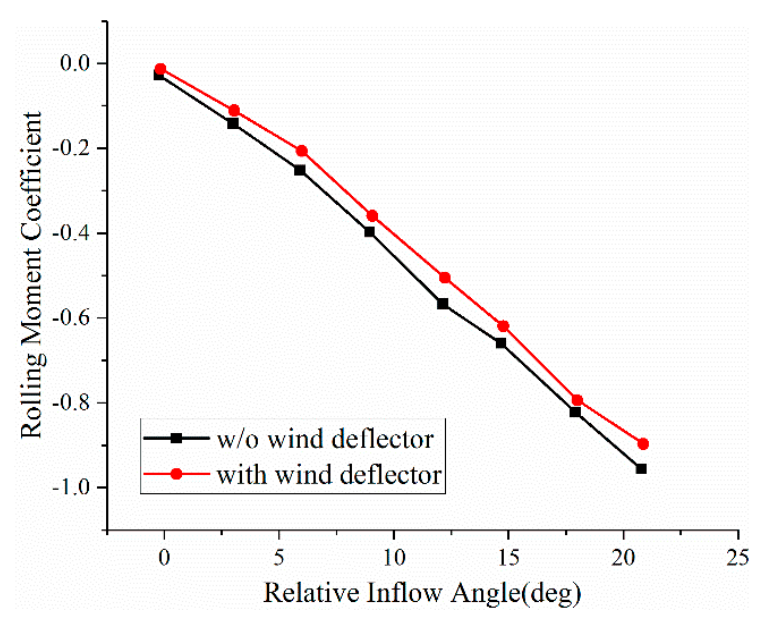

Figure 16. Comparison of rolling moment coefficient.

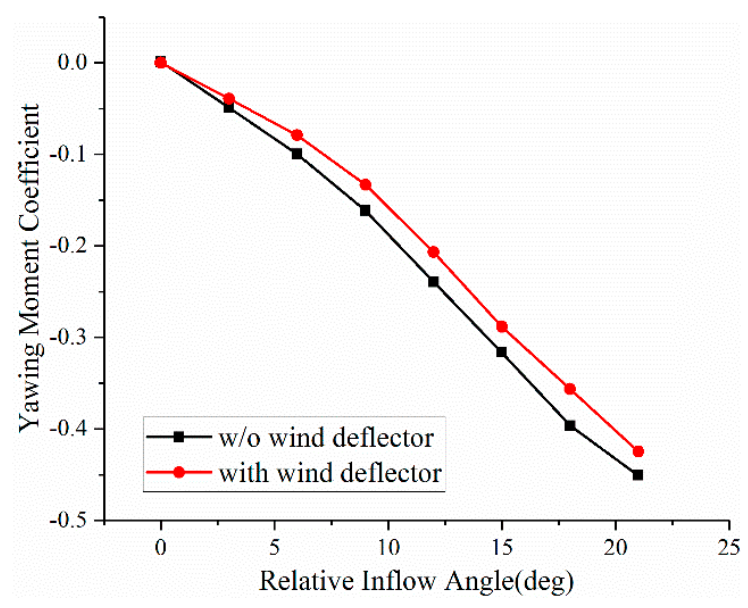

Figure 17. Comparison of yawing moment coefficient.

Figure 18 compares the lateral displacement, lateral acceleration and yaw rate of the heavy tractor semi-trailer with and without a wind deflector. It is shown in the results that the tendencies of these parameters were quite similar, but the lateral displacement, lateral acceleration and yaw rate were reduced accordingly with the wind deflector added, that is, the handling stability was improved. Therefore, it could be concluded that the wind deflector not only could improve the aerodynamic characteristics, but also could enhance the handling stability under crosswind. Comparing the 
two results, the lateral acceleration and yaw rate were decreased by a maximum of $14.6 \%$ and $16.5 \%$, respectively.

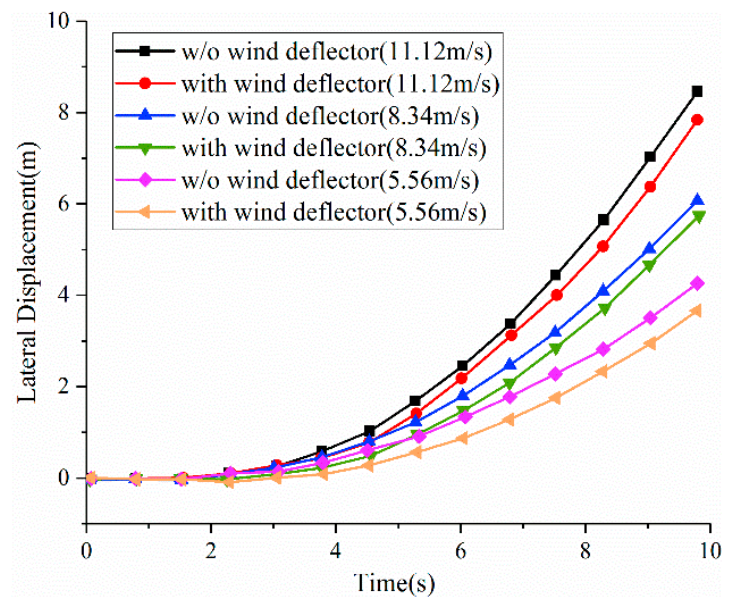

(a)

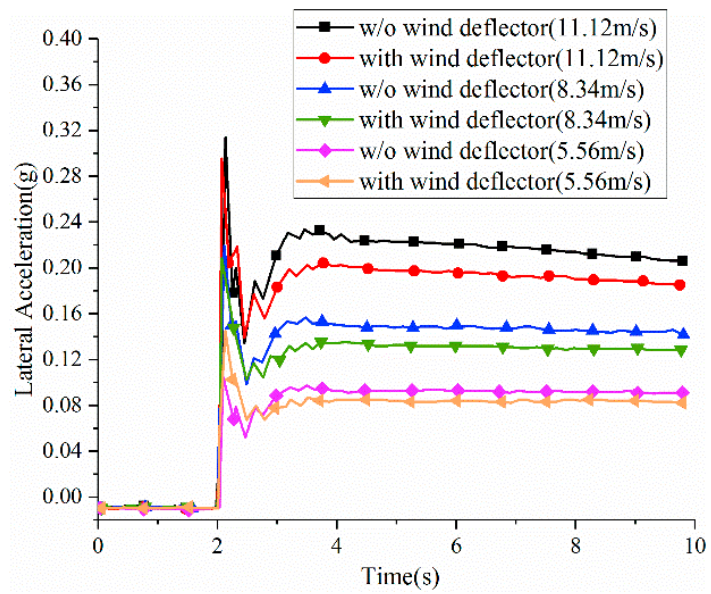

(b)

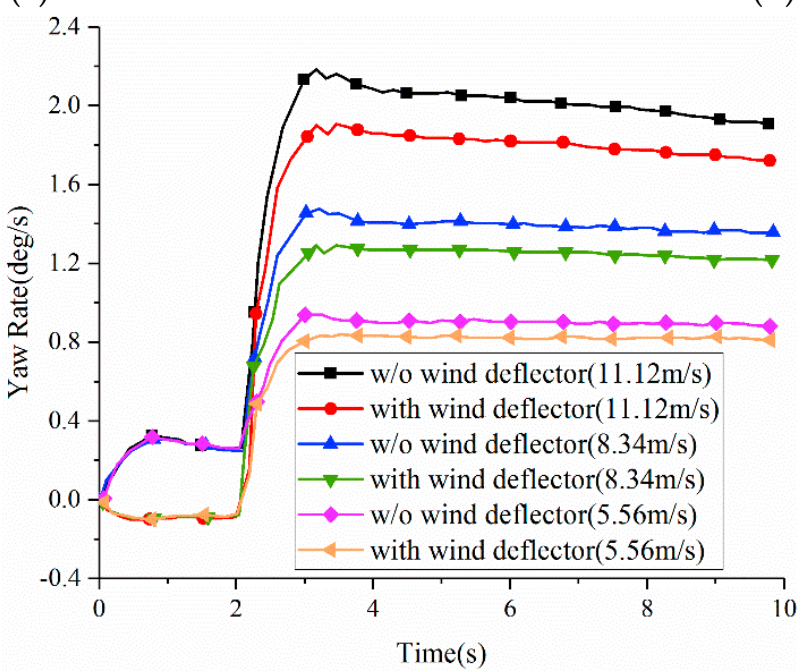

(c)

Figure 18. Comparison of lateral displacement, lateral acceleration and yaw rate with or without wind deflector: (a) lateral displacement; (b) lateral acceleration; (c) yaw rate.

\section{Conclusions}

In the present work, the effect of aerodynamics on the motion of a tractor semi-trailer subjected to crosswinds was analyzed. A coupling method was used to analyze the effects of crosswind on the handling stability of a tractor semi-trailer. In order to obtain the crosswind aerodynamic characteristics, the steady aerodynamics of a tractor semi-trailer were analyzed under various relative inflow angles. To get the mathematical relationship of aerodynamic characteristics under crosswind, a polynomial was used to fit the variation of the aerodynamic coefficient with relative inflow angles. The aerodynamic coefficient functions of the tractor semi-trailer were applied to the aerodynamic module in multi-body dynamic simulation to describe the aerodynamic changes of the vehicle under crosswind. Then, a series of dynamic performance responses including the lateral displacement, the lateral acceleration and the yaw rate were obtained. The dynamic responses, which considered the change of aerodynamics, showed that the crosswind had great influence on the handling stability of the tractor semi-trailer. The handling stability of the tractor semi-trailer with and without a deflector was compared. The dynamic responses indicated that the handling stability of the tractor semi-trailer has been improved a lot with a wind deflector acting on the roof, in which, the lateral acceleration and yaw rate were 
decreased by a maximum of $14.6 \%$ and $16.5 \%$, respectively. The deflector cannot only improve the aerodynamic characteristics, but also the handling stability of the vehicle, which offers a guidance to practical engineering applications. Besides that, the crosswind with a long period of non-uniform changes can promote more significant changes; this is worth improving in future studies.

Author Contributions: Project administration, C.S., C.Z. and Y.W.; Resources, Y.Z.; Validation, J.D.; Writing-original draft, Q.Z. All authors have read and agreed to the published version of the manuscript.

Funding: The research was supported by the National Natural Science Foundation of China (Grant No. 51775395), State's Key Project of Research and Development Plan (Grant No. 2018YFB0105301), the Fundamental Research Funds for the Central Universities (WUT: 2017II18XZ), Innovative Research Team Development Program of Ministry of Education of China (IRT_17R83), 111 Project (B17034) and the China Scholarship Council (No.201906950068).

Conflicts of Interest: The authors declare that they have no conflict of interest. This article does not contain any studies with human participants or animals performed by any of the authors. Informed consent was obtained from all individual participants included in the study.

\section{References}

1. Chu, C.; Chang, C.; Huang, C.; Wu, T.; Wang, C.; Liu, M. Windbreak protection for road vehicles against crosswind. J. Wind Eng. Ind. Aerodyn. 2013, 116, 61-69. [CrossRef]

2. Cooper, K.; Watkins, S. The Unsteady Wind Environment of Road Vehicles. Part One: A Review of The on-Road Turbulent Wind Environment. SAE Paper 2007-01-1236. Available online: https://www.sae.org/ publications/technical-papers/content/2007-01-1236/ (accessed on 16 April 2017).

3. Baker, C. Risk Analysis of Pedestrian and Vehicle Safety in Windy Environments. J. Wind Eng. Ind. Aerodyn. 2015, 147, 283-290. [CrossRef]

4. Mayer, J.; Schrefl, M.; Demuth, R. On Various Aspects of the Unsteady Aerodynamic Effects on Cars Under Crosswind Conditions. SAE Paper 2007-01-1548. Available online: https://www.sae.org/publications/ technical-papers/content/2007-01-1548/ (accessed on 16 April 2017).

5. Ferrand, V. Forces and Flow Structures on a Simplified Car Model Exposed to an Unsteady Harmonic Crosswind. J. Fluids Eng. 2014, 136, 011101-8. [CrossRef]

6. D’Hooge, A.; Rebbeck, L.; Palin, R.; Murphy, Q.; Gargoloff, J.; Duncan, B. Application of Real-World Wind Conditions for Assessing Aerodynamic Drag for On-Road Range Prediction. SAE Paper 2015-01-1551. Available online: https://www.sae.org/publications/technical-papers/content/2015-01-1551/ (accessed on 14 April 2015).

7. Tunay, T.; Firat, E.; Sahin, B. Experimental investigation of the flow around a simplified ground vehicle under effects of the steady crosswind. Int. J. Heat Fluid Flow 2018, 71, 137-152. [CrossRef]

8. Gajendra Singh, M.; Nagpurwala, Q.; Nassar, A.; Shankapal, S. Numerical Investigations on Crosswind Aerodynamics and Its Effect on the Stability of a Passenger Car. SAE Paper 2009-26-059. Available online: https://www.sae.org/publications/technical-papers/content/2009-26-0059/ (accessed on 21 January 2009).

9. Tsubokura, M.; Nakashima, T.; Ikenaga, T.; Onishi, K.; Kitoh, K.; Oshima, N.; Kobayashi, T. HPC-LES for the Prediction of Unsteady Aerodynamic Forces on a Vehicle in a Gusty Cross-Flow Condition. SAE Paper 2008-01-3001. Available online: https://www.sae.org/publications/technical-papers/content/2008-01-3001/ (accessed on 2 December 2008).

10. Cai, C.; Chen, S. Framework of Vehicle-Bridge-Wind Dynamic Analysis. J. Wind Eng. Ind. Aerodyn. 2004, 92, 579-607. [CrossRef]

11. Kee, J.; Rho, J.; Kim, K.; Lee, D. High Speed Driving Stability of Passenger Car Under Crosswind Effects. Int. J. Automot. Technol. 2014, 15, 741-747. [CrossRef]

12. Fujihashi, K.; Okumura, K. Analysis of Vehicle Stability in Crosswinds. SAE Paper 2000-05-0255. Available online: https://www.sae.org/publications/technical-papers/content/2000-05-0255/?src=942507 (accessed on 12 June 2000).

13. Nakashima, T.; Tsubokura, M.; Ikenaga, T.; Doi, Y. HPC-LES for Unsteady Aerodynamics of a Heavy Duty Truck in Wind Gust-2nd report: Coupled Analysis with Vehicle Motion. SAE Paper 2010-01-1021. Available online: https://www.sae.org/publications/technical-papers/content/2010-01-1021/ (accessed on 12 April 2010). 
14. Juhlin, M.; Eriksson, P. A Vehicle Parameter Study on Crosswind Sensitivity of Buses. SAE Paper 2004-01-2612. Available online: https://www.sae.org/publications/technical-papers/content/2004-01-2612/ (accessed on 26 October 2004).

15. Schroeck, D.; Krantz, W.; Widdecke, N.; Wiedemann, J. Unsteady Aerodynamic Properties of a Vehicle Model and Their Effect on Driver and Vehicle under Side Wind Conditions. SAE Paper 2011-01-0154. Available online: https://www.sae.org/publications/technical-papers/content/2011-01-0154/ (accessed on 12 April 2011).

16. Tsubokura, M.; Takahashi, K.; Matsuuki, T.; Nakashima, T.; Ikenaga, T.; Kitoh, K. HPC-LES for Unsteady Aerodynamics of a Heavy Duty Truck in Wind Gust-1st report: Validation and Unsteady Flow Structures. SAE Paper 2010-01-1010. Available online: https://www.sae.org/publications/technical-papers/content/201001-1010/ (accessed on 12 April 2010).

17. Spalding, D.B. Chemical reaction in turbulent fluids. J. Phys. Chem. Hydrodyn. 1983, 4, 323-336.

18. Karabelas, S.J.; Koumroglou, B.C.; Argyropoulos, C.D.; Markatos, N.C. High Reynolds number turbulent flow past a rotating cylinder. Appl. Math. Modell. 2012, 36, 379-398. [CrossRef]

19. Launder, B.E.; Spalding, D.B. Mathematical Models of Turbulence; Academic Press: London, UK, 1972.

20. Reynolds, W.; Cebeci, T. Calculation of turbulent flows. In Ch. 5 in Turbulence. Part of the Topics in Applied Physics Book Series, 2nd ed.; Bradshaw, P., Ed.; Springer: Berlin, Germany, 1978; Volume 12, pp. 193-229.

21. Badshaw, P. Introduction to turbulence. In Ch. 1 in Turbulence. Topics in Applied Physics, 2nd ed.; Bradshaw, P., Ed.; Springer: Berlin, Germany, 1978; Volume 12, pp. 1-44.

22. Launder, B.E.; Sharma, B.I. Application of the energy dissipation model of turbulence to the calculation of flow near a spinning disk, Lett. Heat Mass Transf. 1974, 1, 131-138. [CrossRef]

23. Kolmogorov, A.N. Equations of turbulent motion of an incompressible fluid. Izv Acad. Nauk SSSR Phys. 1942, 6, 56-58.

24. Wilcox, D.C. Reassessment of the scale-determining equation for advanced turbulence models. AIAA J. 1988, 26, 1299-1310. [CrossRef]

25. Menter, F.R. Two-equation eddy-viscosity turbulence models for engineering applications. AIAA J. 1994, 32, 1598-1605. [CrossRef]

26. Yakhot, V.; Orszag, S.A.; Thangam, S.; Gatski, T.B.; Speziale, C.G. Development of turbulence models for shear flows by a double expansion technique. Phys. Fluids Fluid Dyn. 1992, 4, 1510-1520. [CrossRef]

27. Koutsourakis, N.; Bartzis, J.G.; Markatos, N.C. Evaluation of Reynolds stress, k-e and RNG k-e turbulence models in street canyon flows using various experimental datasets. Environ. Fluid Mech. 2012, 12, 379-403. [CrossRef]

28. Argyropoulos, C.D.; Sideris, G.M.; Christolis, M.N.; Nivolianitou, Z.; Markatos, N.C. Modelling pollutants dispersion and plume rise from large hydrocarbon tank fires in neutrally stratified atmosphere. Atmos. Environ. 2010, 44, 803-813. [CrossRef]

29. Han, Z.; Reitz, R.D. Reitz. Turbulence modeling of internal combustion engines using RNG $k-\varepsilon$ models. Combust. Sci. Technol. 1995, 106, 267-295. [CrossRef]

30. Ahmadi-Befrui, B.; Gosman, A.D. Assessment of variants of the $k-\epsilon$ turbulence model for engine flow applications. Int. J. Numer. Methods Fluids 1989, 9, 1073-1086. [CrossRef]

31. Wang, Y.; Li, S.; Xie, X.; Deng, Y.; Liu, X.; Su, C. Performance evaluation of an automotive thermoelectric generator with inserted fins or dimpled-surface hot heat exchanger. Appl. Energy 2018, 218, 391-401. [CrossRef]

32. Wang, Y.; Li, S.; Yang, X. Numerical investigation of the passive control of cavity flow oscillations by a dimpled non-smooth surface. Appl. Acoust. 2016, 111, 16-24. [CrossRef]

33. Gong, X.; Gu, Z.; Song, X.; Wang, Y. Aerodynamic Shape Optimization of a Container-Truck's Wind Deflector Using Approximate Model. SAE Paper 2010-01-2035. Available online: https://www.sae.org/publications/ technical-papers/content/2010-01-2035/ (accessed on 5 October 2010).

34. Yang, Z.G.; Johnson, J.P.; Morley, J.B.; Unaune, S.; Sovani, S.D. Dynamic Moving Mesh CFD Study of Semi-truck Passing a Stationary Vehicle with Hood Open. SAE Paper 2007-01-0111. Available online: https://www.sae.org/publications/technical-papers/content/2007-01-0111/ (accessed on 16 April 2007).

35. Gong, X.; Gu, Z.Q.; Li, Z.L. A Study on the Numerical Simulation of Car Aerodynamic Characteristics Under Crosswind Conditions. Automot. Eng. 2010, 32, 13-17.

36. Lun, T.; Savas, O. Transient Aerodynamics of Vehicle Platoons during in-line Oscillations. J. Wind Eng. Ind. Aerodyn. 2001, 89, 1085-1111. 
37. Osth, J.; Krajnovic, S. A Study of the Aerodynamics of a Generic Container Freight Wagon using Large-Eddy Simulation. J. Fluids Struct. 2014, 44, 31-51. [CrossRef]

38. García, J.; Muñoz-Paniagua, J.; Jiménez, A.; Migoya, E.; Crespo, A. Numerical Study of the Influence of Synthetic Turbulent Inflow Conditions on the Aerodynamics of a Train. J. Fluids Struct. 2015, 56, 134-151. [CrossRef]

39. Zhu, L.; Li, L.; Xu, Y.; Zhu, Q. Wind Tunnel Investigations of Aerodynamic Coefficients of Road Vehicles on Bridge Deck. J. Fluids Struct. 2012, 30, 35-50. [CrossRef]

40. Hou, Y.; Ahmadian, M. Effects of Commercial Truck Configuration on Roll Stability in Roundabouts. SAE Paper 2015-01-2741. Available online: https:/www.sae.org/publications/technical-papers/content/2015-012741/ (accessed on 29 September 2015).

C 2020 by the authors. Licensee MDPI, Basel, Switzerland. This article is an open access article distributed under the terms and conditions of the Creative Commons Attribution (CC BY) license (http://creativecommons.org/licenses/by/4.0/). 